\title{
Db-Igs - eine Plattform zur Herstellung multispezifischer Antikörper
}

OLIVER SEIFERT, ROLAND E. KONTERMANN

INSTITUT FÜR ZELLBIOLOGIE UND IMMUNOLOGIE, UNIVERSITÄT STUTTGART

\section{$\mathrm{Bi}$ - and multispecific antibodies are designated as the next generation of antibody-based therapy, not least because of their wide therapeuti- cal applications, in particular in the field of immuno-oncology. Here, we present the $\mathrm{Db}$-lg platform enabling the generation of a variety of IgG-like molecules with varying valency and specificity with great potentials to generate therapeutic antibodies.}

DOI: $10.1007 / \mathrm{s} 12268-020-1376-4$

(C) Die Autoren 2020
Antikörper revolutionierten in den letzten Jahrzehnten die Therapie vieler unterschiedlicher Krankheiten, wie z. B. Krebs oder chronisch-entzündliche Erkrankungen. Diese klassischen monoklonalen Antikörper sind monospezifisch (sie binden an ein Antigen) und meist bivalent (sie besitzen zwei Antigenbindestellen) und werden therapeutisch oft als Antagonisten (Inhibitoren) ein- gesetzt. Trotz vielfältiger Einsatzgebiete sind diese monoklonalen Antikörper in ihrer therapeutischen Anwendung limitiert. Diese Limitierung ist einerseits gegeben durch die Monospezifität der monoklonalen Antikörper und andererseits durch die zu behandelnden Krankheiten, die meist durch mehrere Fakbi- und multispezifischen Antikörpern in den toren bedingt sind. Mit der Entwicklung von

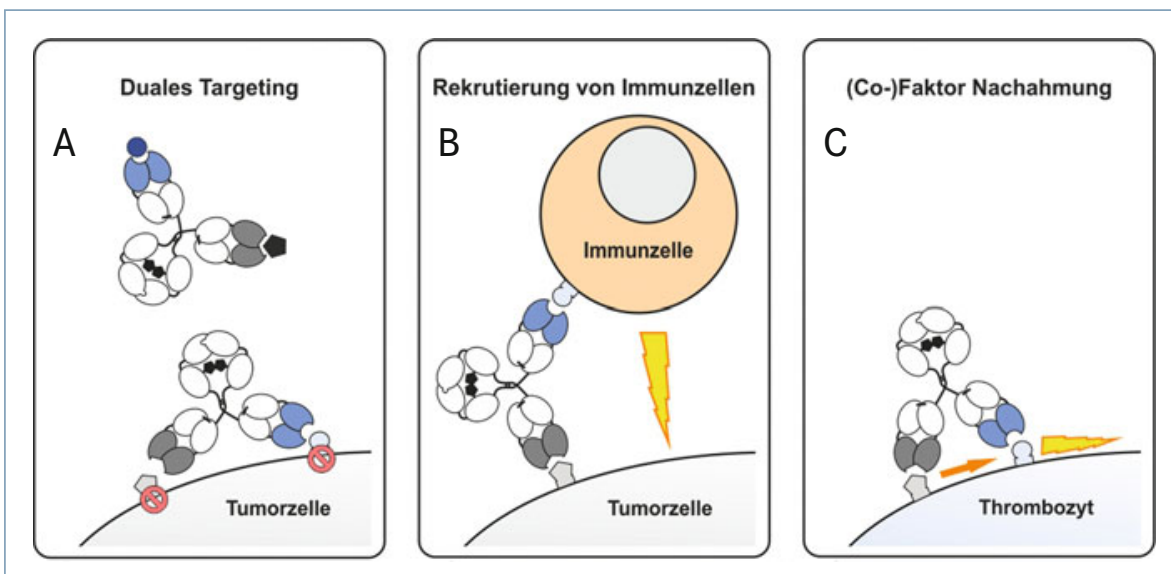

$\Delta$ Abb. 1: Anwendungsbeispiele von therapeutischen bispezifischen Antikörpern. A, Beim dualen Targeting wird z. B. die Signaltransduktion von Tumorzellen durch Bindung und Inhibierung mehrerer Oberflächenrezeptoren oder Liganden moduliert. B, Für die Rekrutierung von Immunzellen (T- oder NK-Zellen) werden Triggermoleküle der Immunzelle (CD3 bei T-Zellen oder CD 16 bei NKZellen) und Antigene der Tumorzelle gleichzeitig gebunden. Dadurch wird die Immunzelle aktiviert und induziert die Lyse der Tumorzelle. Der für die Behandlung von B-Zell-Leukämie zugelassene Blincyto nutzt das Retargeting von T-Zellen als Wirkmechanismus. C, Bei der (Ko-)Faktor-Nachahmung bewirkt der bispezifische Antikörper das Zusammenbringen von zwei gebundenen Faktoren. Hemlibra wird als Ersatz für den Gerinnungsfaktor VIIla für die Behandlung der Hämophilie A eingesetzt und bewirkt das Zusammenlagern der Gerinnungsfaktoren IXa und X. letzten Jahren wurde die nächste Generation therapeutischer Antikörper auf den Weg gebracht. Die Anwendungen von solchen biund multispezifischen Antikörpern decken ein breites Spektrum an neuen Wirkmechanismen ab. Ihre primäre therapeutische Anwendung lag zunächst in der Rekrutierung von Immunzellen (T-Zellen) für die Krebstherapie, welche mit monoklonalen Antikörpern nicht möglich ist. Dies macht die Verwendung von bispezifischen Antikörpern im Kontext der Rekrutierung von Immunzellen obligatorisch. Mittlerweile wurden in den vergangenen Jahrzehnten weitere Strategien für die Therapie mit bi- und multispezifischen Antikörpern entwickelt (Abb. 1). Beispielsweise werden durch das duale Targeting gleichzeitig zwei bzw. mehrere krankheitsbedingende Faktoren erkannt und deren Aktivität neutralisiert. Im optimalen Fall besitzen die bi- und multispezifischen Antikörper eine bessere therapeutische Wirkung (synergistischer Effekt) als die Kombination der monoklonalen Antikörper. Aber auch ein Nachahmen von fehlenden oder defekten Faktoren oder Kofaktoren ist durch bispezifische Antikörper möglich. Zugelassene bispezifische Antikörper sind Blincyto (Blinatumomab; Amgen) für die Rekrutierung von T-Zellen für die Tumortherapie und Hemlibra (Emicizumab; Roche) als Ersatz für den Gerinnungsfaktor VIIIa für die Behandlung der Hämophilie A; beide sind obligatorisch wirkende bispezifische Antikörper [1, 2].

\section{Herstellung bispezifischer Antikörper} Bi- bzw. multispezifische Antikörper sind nicht in der Natur vorzufinden und müssen deshalb im Labor künstlich hergestellt werden. Hier sind ganz unterschiedliche Konstruktionen möglich, die sich in der Größe, der Anzahl der Bindestellen und der molekularen Komposition teilweise stark unterscheiden. Das Spektrum an bispezifischen Antikörperformaten reicht von kleinen Molekülen, die nur aus den variablen Domänen eines Antikörpers aufgebaut sind (wie z. B. Blincyto), über Moleküle, die eine Immunglobulin-G(IgG)-Struktur besitzen (wie z. B. 
Hemlibra), bis hin zu komplexen Molekülen, bei denen z. B. zusätzliche Bindestellen mit einem IgG-Molekül kombiniert werden [3].

Y-förmige, bispezifische IgG-Antikörper besitzen einen asymmetrischen Aufbau, bestehend aus zwei unterschiedlichen schweren und leichten Ketten. Die korrekte Assemblierung solcher Moleküle in einer Zelle wird allerdings durch die zufällige Paarung der beiden schweren Ketten (heavy chain problem) und der leichten und schweren Ketten (light chain problem) erschwert. Aufgrund der kombinatorischen Diversität bei der Kettenpaarung ist nur jedes zehnte produzierte Molekül funktionell. Dies verringert die Produktivität und erschwert die Aufreinigung des gewünschten Antikörpers. Aus diesem Grund wurden für die Herstellung von asymmetrischen Molekülen unterschiedliche Strategien entwickelt, welche die korrekte Kettenassemblierung favorisieren und Fehlpaarungen verringern. Beispielsweise wurden durch gezieltes Einfügen von Mutationen in den Fc-Teil (z. B. knob-into-holeTechnologie [kih]) bzw. in den Fab-Arm heterodimerisierende Varianten entwickelt, um das heavy bzw. light chain problem zu lösen.

Symmetrische Moleküle werden meist durch die Fusion zusätzlicher Antigenbindestellen an ein monospezifisches Antikörpermolekül generiert. Für die Herstellung dieser Moleküle ist eine Modifikation des Fc-Teils bzw. des Fab-Arms nicht notwendig. Beispiele hierfür sind unter anderem tetravalente Moleküle, die jeweils zwei Antigenbindestellen für zwei unterschiedliche Antigene besitzen (scFv-Fc, DVD-Ig und CODV-Ig). Diese Formate besitzen jedoch oft eine geringe Stabilität, eine eingeschränkte Antigenbindung und/oder mehrere und teilweise lange Linker für die Verbindung der unterschiedlichen Antigenbindestellen.

\section{Diabody-Ig-Technologie}

Unsere Arbeitsgruppe entwickelte in den letzten Jahren eine neue modulare Plattform für die Herstellung von multivalenten und multispezifischen Antikörper-ähnlichen Molekülen: die Diabody-Ig-Technologie (Db-Ig; Abb. 2). Die Bindeeinheit der Moleküle dieser Plattform wird durch einen Diabody (Db) gebildet. Hierbei handelt es sich um ein dimeres Modul mit zwei gleichen oder unterschiedlichen Bindestellen. Diese Dbs bestehen aus der variablen Domäne der schweren Kette $\left(\mathrm{V}_{\mathrm{H}}\right)$ und der variablen Domäne der leichten Kette $\left(\mathrm{V}_{\mathrm{L}}\right)$, die durch einen

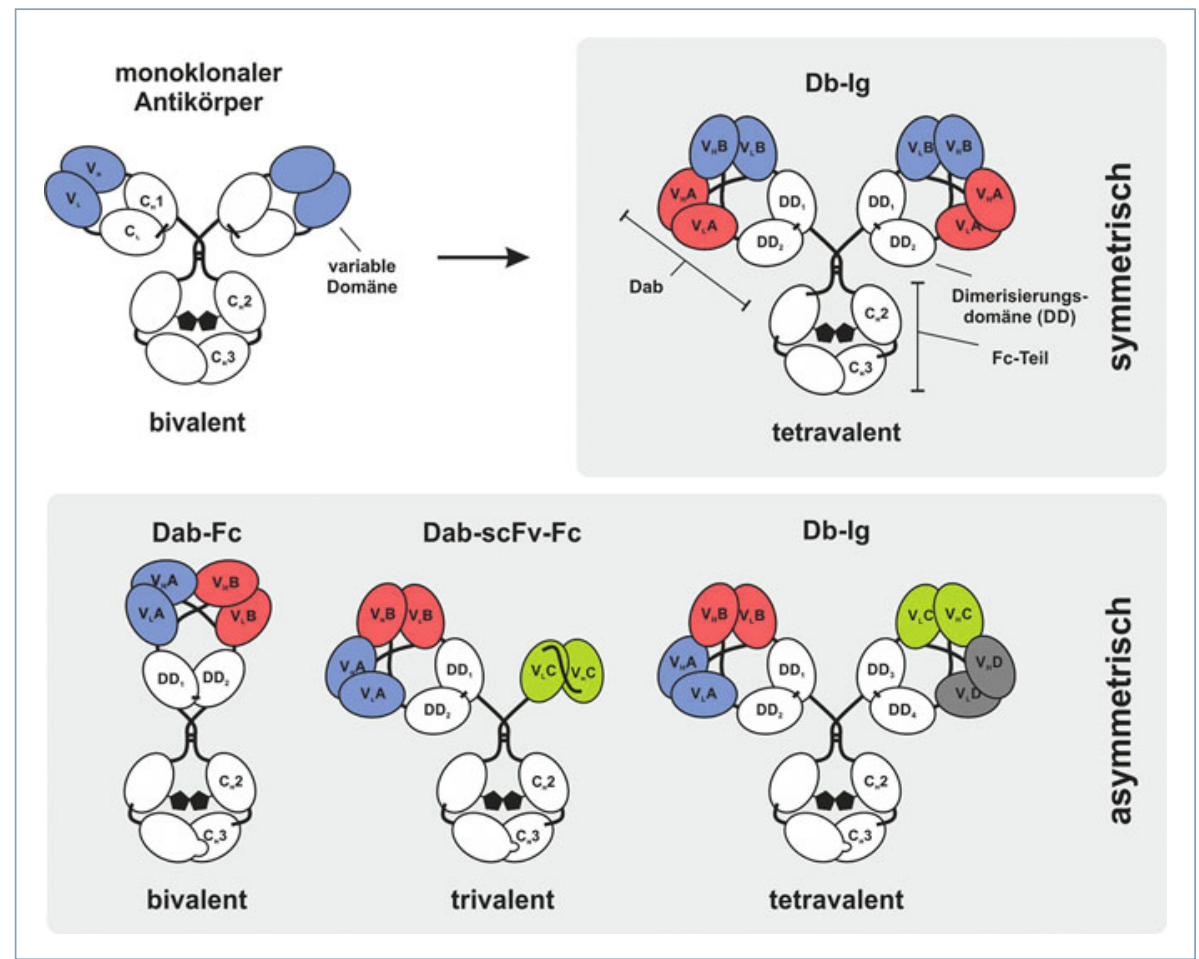

A Abb. 2: Die Plattform Diabody-lg (Db-lg). Die variablen Domänen monoklonaler Antikörper dienen als Basis für die Db-Ig-Plattform. Durch die Fusionierung einer Diabody-Einheit, bestehend aus den variablen Domänen eines oder mehrerer Antikörper, und einer Dimerisierungsdomäne entsteht die Dab-Einheit (Diabody-Fab). Durch die weitere Fusionierung an einen homodimerisierenden Fc-Teil können symmetrische tetravalente Moleküle hergestellt werden, die mono- oder bispezifisch (hier gezeigt) sind. Durch die Verwendung eines heterodimerisierenden Fc-Teils können asymmetrische Moleküle mit unterschiedlicher Valenz (bi-, tri- und tetravalent) und Spezifität (mono-, bi-, tri- und tetraspezifisch) hergestellt werden.

kurzen Linker von wenigen Aminosäuren verbunden sind $\left(\mathrm{V}_{\mathrm{H}}\right.$-Linker- $\left.\mathrm{V}_{\mathrm{L}}\right)$ und ein Dimer bilden. Diese Db-Einheit wird durch die Fusionierung an Dimerisierungsdomänen weiter stabilisiert, ein Schlüsselmerkmal dieser Technologie. Hierfür verwenden wir konstante Domänen aus natürlichen Immunglobulinen, die entweder homodimerisieren oder heterodimerisieren. Für die Homodimerisierung eignen sich die beiden zweiten konstanten Domänen aus IgM (MHD2: IgM constant heavy chain domain 2) [4] und IgE (EHD2: IgE constant heavy chain domain 2) [5]. Dagegen können $\mathrm{C}_{\mathrm{H}} 1 / \mathrm{C}_{\mathrm{L}}$ des IgGs oder eine modifizierte Variante von EHD2 (hetEHD2) als Heterodimerisierungsmodule eingesetzt werden. Aufgrund der strukturellen Gegebenheiten kann die Diabody-Einheit direkt an die Dimerisierungsdomäne fusioniert und auf das Einfügen von zusätzlichen Linkern verzichtet werden. Diese DiabodyFab-Moleküle (Dab) sind bivalent und monobzw. bispezifisch. Durch eine weitere Fusionierung an einen Fc-Teil können multivalente Db-Ig-Moleküle hergestellt werden. Abhängig vom verwendeten Fc-Teil (homo- bzw. heterodimerisierend) lassen sich so symmetrische tetravalente, mono- oder bispezifische bzw. asymmetrische Db-Ig-Moleküle mit zwei, drei oder vier Antigenbindestellen (Valenzen) und unterschiedlicher Spezifität bilden (Abb. 2).

\section{Tetravalente, bispezifische Db-lg- Moleküle}

Wir untersuchten sowohl die Herstellbarkeit als auch die therapeutischen und pharmazeutischen Eigenschaften eines tetravalenten, bispezifischen Moleküls der Db-IgPlattform [6]. Dazu entwickelten wir ein symmetrisch aufgebautes Db-Ig-Molekül, das jeweils zwei Antigenbindestellen für die Tumor-assoziierten Rezeptor-Tyrosinkinasen EGFR (epidermal growth factor receptor) und HER3 besitzt. Hierfür verwendeten wir die humanisierten variablen Domänen eines zugelassenen Anti-EGFR-Antikörpers (Cetuximab: hu225) und die variablen Domänen eines in unserem Labor entwickelten humanen Anti-HER3-Antikörpers (IgG 3-43) [7]. Ein Strukturmodell verdeutlicht, dass in diesen Molekülen alle vier Bindestellen zugäng- 


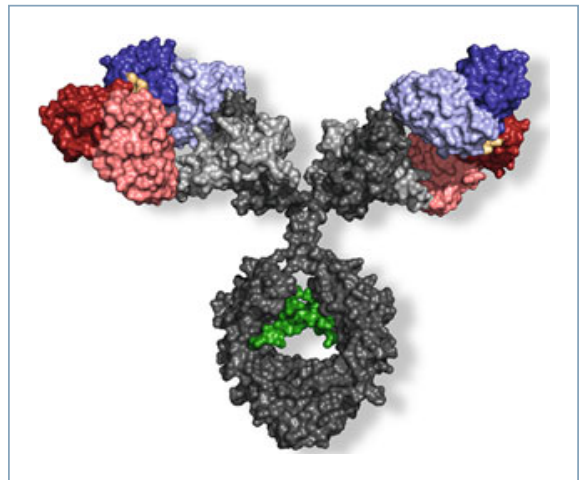

Abb. 3: Strukturmodell eines tetravalenten bispezifischen Db-Ig-Moleküls. Die Kristallstruktur eines Diabody-Moleküls $\left(\mathrm{V}_{\mathrm{H}}\right.$ und $V_{L}$ gegen Antigen 1 bzw. 2 sind in dunkelblau/hellblau bzw. in dunkelrot/hellrot dargestellt; PDB-Code: 1LMK [8]) wurde mit den konstanten Domänen (dunkelgrau: schwere Kette; hellgrau: leichte Kette) eines IgGMoleküls kombiniert. Die Linker in den Diabody-Einheiten sind beige und die Zuckerreste im Fc-Teil grün dargestellt.

lich sind und das Molekül eine IgG-ähnliche Struktur aufweist (Abb. 3). Insgesamt wurden vier verschiedene Db-Ig-Moleküle unter der Verwendung der unterschiedlichen Dimerisierungsdomänen $\left(\mathrm{C}_{\mathrm{H}} 1 / \mathrm{C}_{\mathrm{L}}\right.$, hetEHD2, EHD2 und MHD2) hergestellt und analysiert. Alle vier Proteine konnten mittels einer EinSchritt Affinitätschromatographie aus dem Zellkulturüberstand transfizierter Zellen mit hoher Reinheit und mit Antikörper-ähnlicher Produktivität aufgereinigt werden. Die Moleküle besaßen nicht nur eine ähnlich gute Bindung wie die parentalen Antikörper, sondern konnten zudem gleichzeitig an beide Zielstrukturen binden. In Zellkulturexperimenten resultierte dies in einer verstärkten Inhibierung der Proliferation und einer ausgeprägten Induktion des programmierten Zelltods (Apoptose) von Tumorzellen. Zudem konnten alle vier Db-Ig-Moleküle in Mengen und einer Reinheit produziert werden, die vergleichbar sind mit den Werten normaler Antikörper. Darüber hinaus ist auch die Serumhalbwertszeit dieser Moleküle in vivo ähnlich derjenigen von IgG-Molekülen. Für die hier analysierten Moleküle der Db-IgPlattform wurden also IgG-ähnliche pharmazeutische Eigenschaften und eine vorteilhaf- te Bioaktivität im Vergleich zu den parentalen Antikörpern nachgewiesen. Dies macht Moleküle auf der Basis der Db-Ig-Plattform interessant für die Herstellung von therapeutischen Molekülen.

\section{Die Db-lg-Plattform auf einen Blick}

Mit der hier beschriebenen Db-Ig-Plattform lässt sich eine Vielzahl an unterschiedlichen Formaten generieren, die sich in der Valenz, Spezifität, Geometrie (Anordnung der Antigenbindestellen) und Größe unterscheiden und damit für viele unterschiedliche Anwendungen geeignet sind. Aufgrund des modularen Aufbaus können hier auch andere Bausteine, wie beispielsweise scFv-Fragmente, Fab-Fragmente oder Cytokine an eine Kette integriert werden und so die Vielfalt der DbIg-Moleküle erweitert werden. Diese Db-IgAntikörper lassen sich wie klassische Antikörper aufreinigen und verfügen über ähnliche funktionelle und pharmazeutische Eigenschaften. Die geringe Anzahl von Linkern, die für die Herstellung der Db-Igs notwendig ist, reduziert dabei die Gefahr einer möglichen neutralisierenden Immunantwort. Die Db-Ig-Plattform bietet somit ein großes Potenzial für die Entwicklung von therapeutischen Molekülen nicht nur für die Krebstherapie, sondern auch für andere Indikationen, wie z. B. chronisch-entzündliche und neurodegenerative Erkrankungen, Infektionen und Erkrankungen des Blutes.

\section{Literatur}

[1] Kontermann RE, Brinkmann U (2015) Bispecific antibodies. Drug Discov Today 20:838-847

[2] Labrijn AE, Janmaat ML, Reichert JM et al. (2019) Bispecific antibodies: a mechanistic review of the pipeline. Nat Rev Drug Discov 18:585-608

[3] Brinkmann U, Kontermann RE (2017) The making of bispecific antibodies. MAbs 9:182-212

[4] Seifert O, Plappert A, Heidel N et al. (2012) The IgM CH2 domain as covalently linked homodimerization module for the generation of fusion proteins with dual specificity. Protein Eng Des Sel 25:603-612

[5] Seifert O, Plappert A, Fellermeier S et al. (2014) Tetravalent antibody-scTRAIL fusion proteins with improved properties. Mol Cancer Ther 13:101-111

[6] Seifert O, Rau A, Beha N et al. (2019) Diabody-Ig: a novel platform for the generation of multivalent and multispecific antibody molecules. MAbs 11:919-929

[7] Schmitt LC, Rau A, Seifert O et al. (2017) Inhibition of HER3 activation and tumor growth with a human antibody binding to a conserved epitope formed by domain III and IV. MAbs 9:831-843

[8] Perisic O, Webb PA, Holliger P et al. (1994) Crystal struc ture of a diabody, a bivalent antibody fragment. Structure 2:1217-1226

\section{Funding: Open Access funding provided by Projekt DEAL.}

Open Access: Dieser Artikel wird unter der Creative Commons Namensnennung 4.0 International Lizenz veröffentlicht, welche die Nutzung, Vervielfältigung, erlaubt, sofern Sie den/die ursprünglichen Autor(en) und die Quelle ordnungsgemäß nennen, einen Link zur Creative Commons Lizenz beifügen und angeben, ob Änderungen vorgenommen wurden. Die in diesem Artikel angeben, ob Änderungen vorgenommen wurden. Die in diesem Artikel enthaltenen Bilder und sonstiges Drittmaterial unterliegen ebenfalls der
genannten Creative Commons Lizenz, sofern sich aus der Abbildungslegend nichts anderes ergibt. Sofern das betreffende Material nicht unter der genannten Creative Commons Lizenz steht und die betreffende Handlung nich nach gesetzlichen Vorschriften erlaubt ist, ist für die oben aufgeführten Weiterverwendungen des Materials die Einwilligung des jeweiligen Rechteinhabers einzuholen. Weitere Details zur Lizenz entnehmen Sie bitte der

Korrespondenzadresse:

Dr. Oliver Seifert

Institut für Zellbiologie und Immunologie Universität Stuttgart

Allmandring 31

D-70569 Stuttgart

oliver.seifert@izi.uni-stuttgart.de

Oliver Seifert
Jahrgang 1984. 2004-2010 Biologiestudium an den Universitäten Hohenheim und
Stuttgart. 2010-2014 Promotion an der Universität Stuttgart. Seit 2014 wissen-
schaftlicher Mitarbeiter in der Abteilung Biomedical Engineering am Institut für
Zellbiologie und Immunologie, Universität Stuttgart.

